\title{
Regulation of Nucleo-Cytoplasmic Transport of the Aryl Hydrocarbon Receptor
}

\author{
Kaname Kawajiri* and Togo Ikuta \\ Research Institute, Saitama Cancer Center, 818 Komuro, Ina-machi, Kitaadachi-gun, Saitama 362-0806, Japan
}

(Received March 1, 2004)

\begin{abstract}
The aryl hydrocarbon receptor (AhR) is a ligand-activated transcription factor that plays a role as an intracellular mediator of the xenobiotic signaling pathway. AhR is a nucleo-cytoplasmic shuttling protein mediated by nuclear localization signals (NLS) and nuclear export signals (NES). Protein kinase C-mediated phosphorylation of NLS inhibits the ligand-dependent nuclear import of AhR, suggesting a two-step mechanism of nuclear import. Cell density regulates AhR intracellular localization, due to modulation of nuclear export activity. The p38 mitogenactivated protein kinase (MAPK)-mediated phosphorylation of NES and its dephosphorylation, regulated by cellcell contact signals, may have pivotal roles in the novel AhR relocalization.
\end{abstract}

Key words — aryl hydrocarbon receptor, nucleo-cytoplasmic translocation, phosphorylation, cell density, nuclear localization signal, nuclear export signal

\section{INTRODUCTION}

Mammalian cells contain a variety of receptors that can bind and respond to toxic chemical compounds present in the environment. They include steroid hormone receptors, orphan nuclear receptors such as the constitutive active receptor (CAR) and steroid and xenobiotic receptor (SXR), and the PERARNT-SIM homology region (PAS) family of the transcription factor aryl hydrocarbon receptor (AhR). 2,3,7,8-Tetrachlorodibenzo- $p$-dioxin (TCDD) is one of the most potent toxic contaminants in the environment; it induces various biological effects on animals, including humans, at low-dose levels. These effects include carcinogenicity, teratogenicity, and immunotoxity, and it is possibly toxic to reproductive health. ${ }^{1,2)}$ At present, two molecular mechanisms of AhR-mediated TCDD toxicity are known: the AhR-AhR nuclear translocator (ARNT) heterodimer binds directly to the xenobiotic-responsive element (XRE) in the promoter of target genes, resulting in the activation of expression of genes such as

\footnotetext{
*To whom correspondence should be addressed: Research Institute, Saitama Cancer Center, 818 Komuro, Ina-machi, Kitaadachi-gun, Saitama 362-0806, Japan. Tel.: +81-48-7221111 (ext. 4620, 4622); Fax: +81-48-722-1739; E-mail: kawajiri @ cancer-c.pref.saitama.jp
}

CYP1A13); or alternatively, it functions in coactivator-like fashion by molecular interaction with nuclear receptors, such as estrogen receptor $\alpha$, without direct binding to the XRE. ${ }^{4)}$ Since liganddependent nuclear import of AhR seems to be the first step in the induction of target genes, elucidation of the molecular mechanism underlying the nuclear translocation is vital for understanding not only TCDD toxicity but also the physiologic function of AhR. In this paper, we briefly review the regulation of nucleo-cytoplasmic transport of the AhR.

\section{Signals Responsible for Intracellular Localization of the AhR}

The nuclear localization signal (NLS)-dependent nuclear import of proteins depends on cytosolic factors, and can be separated into two steps. The first step is an energy- and temperature-independent targeting of the nuclear pore mediated by importins $\alpha$ and $\beta$; the second step is an energy- and temperature-dependent nuclear translocation through the nuclear pore mediated by Ran-GDP and $\mathrm{p} 10 .^{5,6)} \mathrm{On}$ the other hand, the nuclear export signal (NES) has been defined as a short sequence of a leucine-enriched amino acid motif, and NES-dependent nuclear export is mediated by chromosome region maintenance 1 (CRM1) in concert with Ran-GTP.

We identified both the NLS and NES of the AhR, 
as shown in Fig. $1{ }^{7)}$ We allocated the AhR-NLS to amino acid residues 13 to 39 , with the bipartite core overlapped by domains of DNA or HSP90 binding. Resistance to TCDD toxicity in mice carrying a mutation in the NLS has recently been reported ${ }^{8}{ }^{8}$ as it has in the AhR-knockout mouse. ${ }^{9)}$ We also identified an AhR-NES composed of amino acid residues 55 to 75 in helix 2, the hydrophobic core involved in heterodimer formation with ARNT. Using these two signals, the AhR shuttles between the cytoplasm and the nucleus of a cell, ${ }^{10)}$ and the shuttling activity is inhibited by leptomycin B (LMB), a specific inhibitor of nuclear export. In addition, the ARNT-NLS in the range from 39 to 61 amino acid residues was also identified. ${ }^{11)}$

\section{Regulation of Nuclear Import of the AhR}

(1) Phosphorylation of AhR-NLS Inhibits LigandDependent Nuclear Import of the AhR

In general, intracellular localization of nucleocytoplasmic shuttling proteins is determined by the balance between nuclear import and export activity. Phosphorylation or dephosphorylation, especially close to the NLS or NES, frequently regulates the intracellular distribution of such proteins, resulting in spatial and temporal specific gene regulation. ${ }^{12)}$ Two protein kinase C (PKC) sites of Ser-12 and Ser-36 are observed one amino acid upstream from each of the two basic amino acid segments of the NLS (Fig. 1), AhR(13-16:RKRR) and AhR(37$39: \mathrm{KRH})$, and we found that the ligand-dependent nuclear import of $\mathrm{AhR}$ is inhibited by substitution of aspartic acid for Ser-12 (S12D) or Ser-36 (S36D), which mimics the negative charge of phosphorylation. This observation was supported by microinjection analysis, an in vitro nuclear transport assay, and luciferase reporter assay. From these results, it is likely that the NLS of AhR constitutes a phosphorylation-mediated regulatory module of nuclear localization, and phosphorylation of NLS appears to lead to a decreased association between NLS and importin $\alpha .{ }^{13)}$

\section{(2) Two-Step Mechanism of Ligand-Dependent Nuclear Import of the AhR}

Mutants of the AhR which mimic the phosphorylated form of AhR-NLS have abrogated nuclear import activity in the presence of ligand, while mutants of the AhR which mimic the dephosphorylated NLS also have no nuclear localization activity without ligand. Based on these results, we can envisage a two-step mechanism of ligand-dependent nuclear

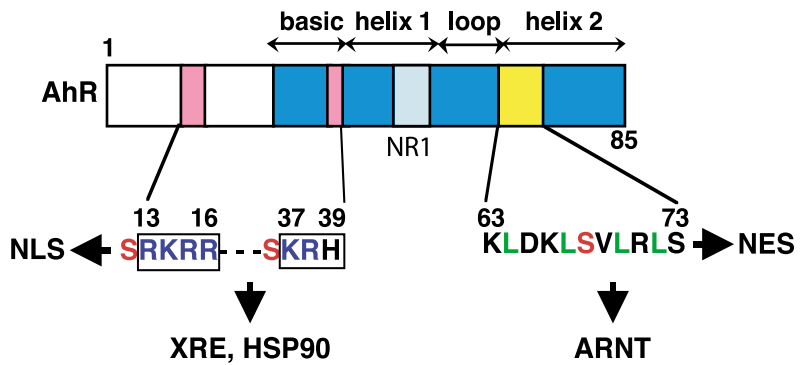

Fig. 1. Schematic Representation of the Functional $\mathrm{NH}_{2}$ Terminal of the Human AhR

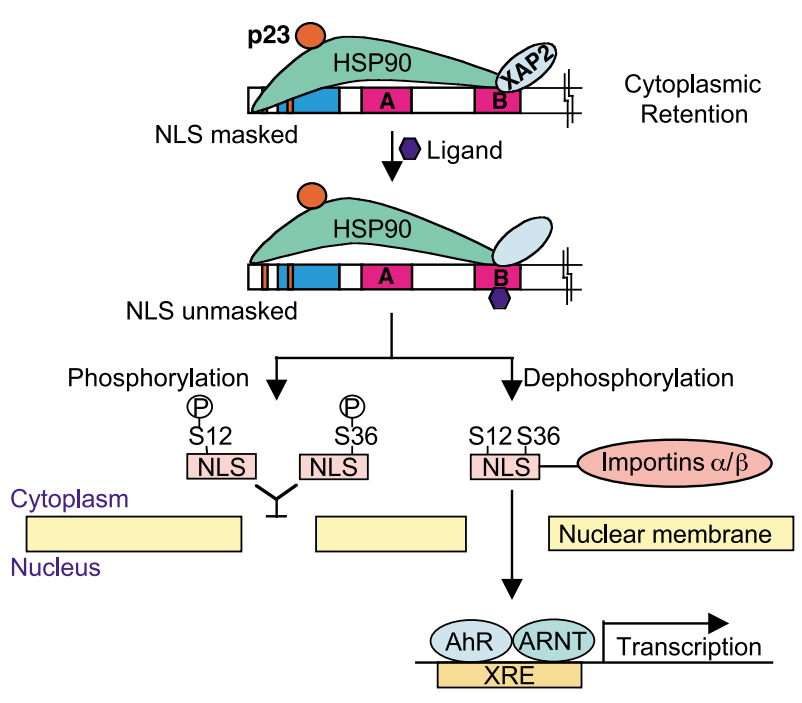

Fig. 2. Two-Step Mechanism of the Ligand-Dependent Nuclear Import of the AhR

import of the AhR, as shown in Fig. $2 .{ }^{13)}$ In the absence of ligand, the AhR is associated with a complex of Hsp90, p23, and XAP2 and resides predominantly in the cytoplasmic compartment. ${ }^{14,15}$ The steric hindrance masking of AhR-NLS through two molecules of Hsp90 is essential for cytoplasmic retention of the AhR. The first step is ligand binding to the AhR, which makes it necessary to facilitate interaction of the NLS with nuclear import components. ${ }^{7,14,15)}$ The second step is the phosphorylationregulated nuclear import process, in which the phosphorylated NLS abrogates the ligand-dependent nuclear import, while the dephosphorylated NLS promotes it sufficiently to interact with NLS receptors, followed by nuclear import. The AhR then rapidly accumulates in the nucleus and is followed by heterodimerization with ARNT for transcription. 


\section{(3) Involvement of the LXXLL-Motif in Subcel- lular Localization of the AhR}

The LXXLL-motif (NR box) has recently been shown to mediate the protein-protein interactions of transcriptional cofactors with nuclear receptors. ${ }^{16)}$ The presence of this motif in the AhR suggested that it might be crucial for the regulation of AhR activity. Mutation of the motif at AhR(50-54) (NR1), which is localized between the NLS and NES in helix 1 (Fig. 1), promotes the efficiency of nuclear localization in the absence of ligand, without altering HSP90 and ARA9 binding or nuclear export activity; another LXXLL-motif at AhR(224-227) (NR2) does not affect the subcellular localization of the AhR. Thus it is likely that the LXXLL-motif of NR1 may contribute to subcellular localization through intra- or intermolecular interactions. ${ }^{17)}$

\section{Regulation of the Nuclear Export of the AhR (1) Cell Density Regulates Intracellular Localiza- tion of the AhR}

Using the keratinocyte cell line $\mathrm{HaCaT}$ and immunohistochemical staining analysis, we found that cell density, but not cell cycle, influences the intracellular distribution of the AhR, with a predominant nuclear localization of the AhR in sparsely distributed cells, localization in both the nucleus and the cytoplasm in subconfluent cells, and predominant cytoplasmic localization in confluent cells. ${ }^{18)}$ Treatment with LMB resulted in increased nuclear accumulation of the AhR even with high cell density, suggesting that the novel AhR relocalization was due to altered nuclear export activity, and that both nuclear import activity and some potential endogenous ligands exist even when cells are confluent. Cell-to-cell communication of the HaCaT cells seems to be crucial in the cytoplasmic localization of the AhR with high cell density, since a clear nuclear localization of the AhR was observed in cells, where $\mathrm{Ca}^{2+}$-dependent cell-to-cell contact was disrupted by a low concentration of $\mathrm{Ca}^{2+}$.

To observe the relation between AhR localization and function, a stable transfectant was isolated by introducing a luciferase gene fused with XRE. When the cells were grown to subconfluence, maximal luciferase activity was observed, but when the cells were further grown to confluence, the luciferase activity was decreased to the basal level. On the other hand, the expression levels of AhR and ARNT did not change under varying conditions of cell density. CYP1A1 was expressed at low cell densities, while involucrine, a specific differentiation marker of keratinocytes, increased gradually with increased cell density. Thus it is likely that the altered XRE-luciferase activity due to cell density resulted from a novel redistribution of the AhR without an altered expression level of the AhR.

In addition, a stable transfectant was also isolated by introducing a plasmid containing green fluoresecent protein (GFP) fused with XRE. The GFP was expressed clearly at low cell density and gradually diminished when the cells grew to confluence or superconfluence. We then carried out an experiment using an in vitro wound-healing model, and the results clearly suggest that XREmediated transcription is activated selectively at the wound edges, where the cells seem to be loosely associated. All of these results support the idea that XRE-mediated transcription is influenced by cell density, and that the AhR/ARNT system functions in this process.

\section{(2) Phosphorylation of AhR-NES Inhibits the Nuclear Export of the AhR}

Since density-dependent AhR relocalization may be due to a change in the regulation of the nuclear export of the AhR, we wanted to determine which amino acid residues of NES were important for the modulation of nuclear export. ${ }^{18)}$ We developed interest in Ser-68 and Ser-73 (Fig. 1), since some transcription factors have Ser or Thr in their NES that is often phosphorylated in both the activated and inactivated forms. When injected into the nucleus, a mutant AhR with S73D retained nuclear export activity as well as the wild type did, while AhR with an S68D mutation was unable to pass through the nuclear pore and remained in the nucleus, suggesting that phosphorylation of Ser-68 inhibits nuclear export.

We produced a peptide antibody against AhR from 61 to 74, containing phosphoserine-68. The Ser68 in the NES of the AhR was phosphorylated not only in the nuclei of livers of 3-methylcholanthrene (MC)-treated mice, but also in confluent $\mathrm{HaCaT}$ cells cultured with Dulbecco's modified Eagle's essential medium (DMEM), followed by further incubation with $\mathrm{Ca}^{2+}$-deficient S-MEM. A single band, which had a slower mobility shift than that of the AhR, was detected by anti-AhR(61-74)-pS68 antibody in cell extracts prepared from S-MEM, and this band was also recognized by the specific antibody to the AhR. These results led us to conclude that Ser-68 in the NES is phosphorylated in the nucleus. 


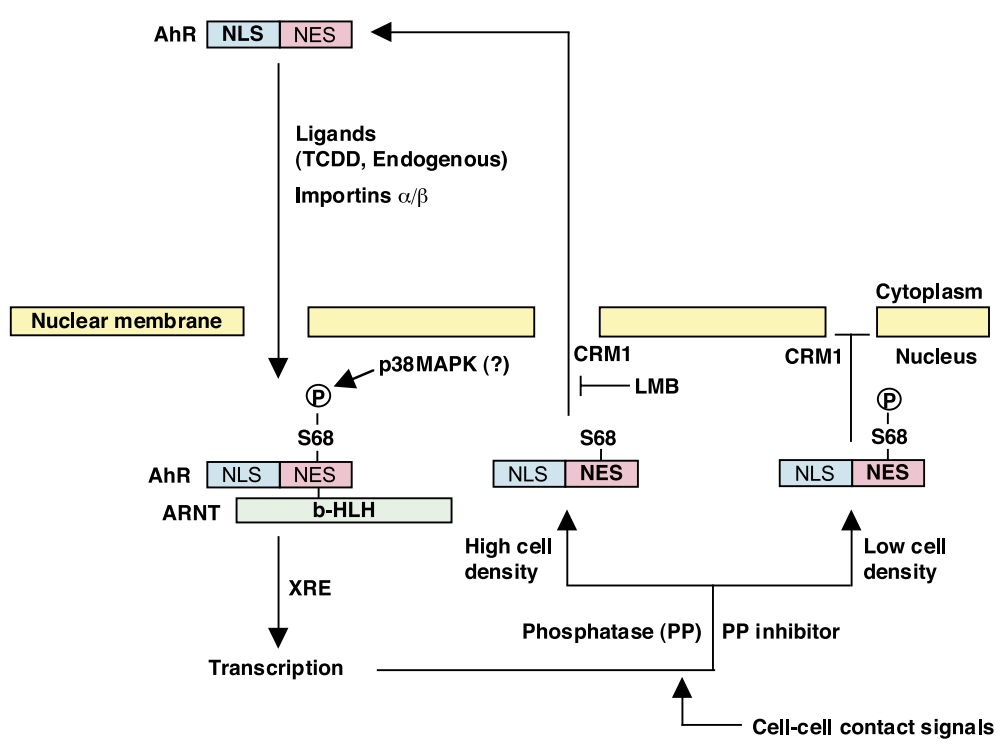

Fig. 3. Proposed Model for Cell Density-Dependent AhR Relocalization

\section{(3) A Model for Cell Density-Dependent AhR Relocalization}

The role of p38 mitogen-activated protein kinase (MAPK)-dependent phosphorylation of the NES in suppressing the nuclear export of the $\mathrm{p} 53^{19)}$ or estrogen receptor $\alpha^{20)}$ has previously been reported. In this context, it is likely that the Ser-68 within the NES is the corresponding amino acid that is modified by the p38 MAPK. If the p38-mediated phosphorylation of AhR-NES participates in the cell density-dependent redistribution of the AhR, exposure to an inhibitor of $\mathrm{p} 38$ would result in altered subcellular localization of the AhR. To test this, cultured $\mathrm{HaCaT}$ cells in the subconfluent state, where AhR is distributed evenly in both the cytoplasm and the nucleus, were treated briefly with SB203580. We then observed a more cytoplasmic distribution of the AhR. In contrast, when treated with U0126, a MAP kinase-extracellular signal-regulated kinase (ERK) kinase (MEK) inhibitor of the ERK-dependent cascade, the subcellular localization of the AhR was not affected, and nuclear retention of AhR mediated by p38 MAPK was also supported by a reporter assay. In addition, we observed that the p38 MAPK was activated by the medium change from DMEM to calcium-deficient S-MEM after only $15 \mathrm{~min}$ with the HaCaT cells, followed by CYP1A1 induction. In contrast, when confluent $\mathrm{HaCaT}$ cells, where the $\mathrm{AhR}$ is distributed in the cytoplasm, were treated briefly with a protein phosphatase inhibitor of okadaic acid, a significant shift toward a more nuclear distribution of the AhR was observed. Thus the novel relocalization of the AhR was made more cytoplasmic by a p38 MAPK inhibitor, and more nuclear by a phosphatase inhibitor, suggesting that p38-dependent phosphorylation and its dephosphorylation may be involved in the novel relocalization of AhR caused by cell density.

In conclusion, it is likely that the cell densitydependent novel relocalization of the AhR can be explained by changes in the regulation of nuclear export which may include both phosphorylation of AhR-NES by p38-dependent MAPK for nuclear retention and its dephosphorylation regulated by cellcell contact signals for nuclear export. The balance of protein phosphatase and its inhibitor may play a pivotal role in regulating the intracellular distribution of AhR. A proposed model ${ }^{18)}$ for AhR relocalization is shown in Fig. 3.

The expression of the AhR was specific to the cell type, organ/tissue, and the developmental stage, and intracellular localization of the protein was altered in a time-specific manner in mice embryos, suggesting that the ligand-activated transcription factor may be important in normal embryonic development. Since the regulated intracellular localization of transcription factor can serve as a biological switch in response to various signals, a novel relocalization of the AhR caused by cell density appears to be an essential event for the biological function of the AhR. Phosphorylation/dephosphorylation-dependent AhR movement in and out of the nucleus provides a simple, reversible, and rapid means to control responses to different environmen- 
tal or physiologic conditions and extracellular signals in cells.

Acknowledgements This work was supported in part by Grants-in-Aid for Scientific Research from the Ministry of Education, Science, Sports and Culture of Japan, Health Sciences Research Grants from the Ministry of Health, Labor and Welfare of Japan, and Core Research for Evolutional Science and Technology, Japan Science and Technology Agency.

\section{REFERENCES}

1) Poland, A. and Knutson, J. C. (1982) 2,3,7,8Tetrachlorodibenzo- $p$-dioxin and related aromatic hydrocarbons: examination of the mechanism of toxicity. Annu. Rev. Pharmacol. Toxicol., 22, 517 554.

2) Schmidt, J. V. and Bradfield, C. A. (1996) Ah receptor signaling pathways. Anпи. Rev. Cell Dev. Biol., 12, 55-89.

3) Matsushita, N., Sogawa, K., Ema, M., Yoshida, A. and Fujii-Kuriyama, Y. (1993) A factor binding to the xenobiotic responsive element (XRE) to P-450 1A1 gene consists of at least two helix-loop-helix proeins, Ah receptor and ARNT. J. Biol. Chem., 268 , 21002-21006.

4) Ohtake, F., Takeyama, K., Matsumoto, T., Kitagawa, H., Yamamoto, Y., Nohara, K., Tohyama, C., Krust, A., Mimura, J., Chambon, P., Yanagisawa, J., FujiiKuriyama, Y. and Kato, S. (2003) Modulation of oestrogen receptor signaling by association with the activated dioxin receptor. Nature (London), $\mathbf{4 2 3}$, 545-550.

5) Mattaj, I. W. and Englmeier, L. (1998) Nucleocytoplasmic transport: the soluble phase. Annu. Rev. Biochem., 67, 265-306.

6) Nigg, E. A. (1997) Nucleocytoplasmic transport: signals, mechanism and regulation. Nature (London), 386, 779-787.

7) Ikuta, T., Eguchi, H., Tachibana, T., Yoneda, Y. and Kawajiri, K. (1998) Nuclear localization and export signals of the human aryl hydrocarbon receptor. $J$. Biol. Chem., 273, 2895-2904.

8) Bunger, M. K., Moran, S. M., Glover, E., Thomae, T. L., Lahvis, G. P., Lin, B. C. and Bradfield, C. A. (2003) Resistance to 2,3,7,8-tetrachlorodibenzo- $p$ dioxin toxicity and abnormal liver development in mice carrying a mutation in the nuclear localization sequence of the aryl hydrocarbon receptor. J. Biol. Chem., 278, 17767-17774.

9) Mimura, J., Yamashita, K., Nakamura, K., Morita, M., Takagi, N. T., Nakao, K., Ema, M., Sogawa, K.,
Yasuda, M., Katsuki, M. and Fujii-Kuriyama, Y. (1997) Loss of teratogenic response to 2,3,7,8tetrachlorodibenzo- $p$-dioxin (TCDD) in mice lacking the $\mathrm{Ah}$ (dioxin) receptor. Genes Cells, 2, 645654.

10) Ikuta, T., Tachibana, T., Watanabe, J., Yoshida, M., Yoneda, Y. and Kawajiri, K. (2000) Nucleocytoplasmic shuttling of the aryl hydrocarbon receptor. $J$. Biochem., 127, 503-509.

11) Eguchi, H., Ikuta, T., Tachibana, T., Yoneda, Y. and Kawajiri, K. (1997) A nuclear localization signal of human aryl hydrocarbon receptor nuclear translocator/hypoxia-inducible factor $1 \beta$ is a novel bipartite type recognized by the two components of nuclear pore-targeting complex. J. Biol. Chem., 272, 17640-17647.

12) Jans, D. A. and Hubner, S. (1996) Regulation of protein transport to the nucleus: Central role of phosphorylation. Physiol. Rev., 76, 651-685.

13) Ikuta, T., Kobayashi, Y. and Kawajiri, K. (2004) Phosphorylation of nuclear localization signal inhibits the ligand-dependent nuclear import of aryl hydrocarbon receptor. Biochem. Biophys. Res. Commun., 317, 545-550.

14) Lees, M. J. and Whitelaw, M. L. (1999) Multiple roles of ligand in transforming the dioxin receptor to an active basic helix-loop-helix/PAS transcription factor complex with the nuclear protein Arnt. Mol. Cell. Biol., 19, 5811-5822.

15) Kazlauskas, A., Sundstrom, S., Poellinger, L. and Pongratz, I. (2001) The hsp90 chaperone complex regulates intracellular localization of the dioxin receptor. Mol. Cell. Biol., 21, 2594-2607.

16) Heery, D. M., Kalkhoven, E., Hoare, S. and Parker, M. G. (1997) A signature motif in transcriptional co-activators mediates binding to nuclear receptors. Nature (London), 387, 733-736.

17) Ikuta, T., Watanabe, J. and Kawajiri, K. (2002) Characterization of the LxxLL motif in the aryl hydrocarbon receptor: effects on subcellular localization and transcriptional activity. J. Biochem., 131, 79-85.

18) Ikuta, T., Kobayashi, Y. and Kawajiri, K. (2004) Cell density regulates intracellular localization of aryl hydrocarbon receptor. J. Biol. Chem., 279, 19209 19216.

19) Zhang, Y. and Xiong, Y. (2001) A p53 aminoterminal nuclear export signal inhibited by DNA damage-induced phosphorylation. Science, 292, 1910-1915.

20) Lee, H. and Bai, W. (2002) Regulation of estrogen receptor nuclear export by ligand-induced and $\mathrm{p} 38$ mediated receptor phosphorylation. Mol.Cell. Biol., 22, 5835-5845. 